\title{
agReg-SNPdb: A Database of Regulatory SNPs for Agricultural Animal Species
}

\author{
Selina Klees ${ }^{1,2, *,+}+\mathbb{D}$, Felix Heinrich ${ }^{1,+}\left(\mathbb{D}\right.$, Armin Otto Schmitt ${ }^{1,2}\left(\mathbb{D}\right.$ and Mehmet Gültas ${ }^{2,3, *}$ \\ 1 Breeding Informatics Group, Department of Animal Sciences, Georg-August University, Margarethe von \\ Wrangell-Weg 7, 37075 Göttingen, Germany; felix.heinrich@uni-goettingen.de (F.H.); \\ armin.schmitt@uni-goettingen.de (A.O.S.) \\ 2 Center for Integrated Breeding Research (CiBreed), Georg-August University, Albrecht-Thaer-Weg 3, \\ 37075 Göttingen, Germany \\ 3 Faculty of Agriculture, South Westphalia University of Applied Sciences, Lübecker Ring 2, \\ 59494 Soest, Germany \\ * Correspondence: selina.klees@uni-goettingen.de (S.K.); gueltas.mehmet@fh-swf.de (M.G.) \\ + These authors contributed equally to this work.
}

check for updates

Citation: Klees, S.; Heinrich, F.;

Schmitt, A.O.; Gültas, M.

agReg-SNPdb: A Database of

Regulatory SNPs for Agricultural

Animal Species. Biology 2021, 10, 790.

https://doi.org/10.3390/

biology10080790

Academic Editor: W. Brad Barbazuk

Received: 12 July 2021

Accepted: 12 August 2021

Published: 17 August 2021

Publisher's Note: MDPI stays neutral with regard to jurisdictional claims in published maps and institutional affiliations.

Copyright: (c) 2021 by the authors. Licensee MDPI, Basel, Switzerland. This article is an open access article distributed under the terms and conditions of the Creative Commons Attribution (CC BY) license (https:// creativecommons.org/licenses/by/ $4.0 /)$.
Simple Summary: Regulatory SNPs (rSNPs) are SNPs located within promoter regions that have a high potential to alter gene expression by changing the binding affinity of transcription factors to their binding sites. Such rSNPs are gaining importance in the life sciences due to their causality for specific traits and diseases. In this study, we present agReg-SNPdb, the first database comprising rSNP data of seven agricultural and domestic animal species: cattle, pig, chicken, sheep, horse, goat, and $\mathrm{dog}$, and made it usable via a web interface.

Abstract: Transcription factors (TFs) govern transcriptional gene regulation by specifically binding to short DNA motifs, known as transcription factor binding sites (TFBSs), in regulatory regions, such as promoters. Today, it is well known that single nucleotide polymorphisms (SNPs) in TFBSs can dramatically affect the level of gene expression, since they can cause a change in the binding affinity of TFs. Such SNPs, referred to as regulatory SNPs (rSNPs), have gained attention in the life sciences due to their causality for specific traits or diseases. In this study, we present agReg-SNPdb, a database comprising rSNP data of seven agricultural and domestic animal species: cattle, pig, chicken, sheep, horse, goat, and dog. To identify the rSNPs, we constructed a bioinformatics pipeline and identified a total of 10,623,512 rSNPs, which are located within TFBSs and affect the binding affinity of putative TFs. Altogether, we implemented the first systematic analysis of SNPs in promoter regions and their impact on the binding affinity of TFs for livestock and made it usable via a web interface.

Keywords: single nucleotide polymorphism; regulatory SNP; transcription factor; transcription factor binding site; gene regulation; database; agricultural animal species; livestock

\section{Introduction}

The transcriptional regulation of gene expression in higher organisms is essential for various biological processes. In contrast to the process of translation, the transcriptional machinery and its regulatory mechanisms are far from being deciphered [1]. These mechanisms are mainly governed by a special class of regulatory proteins, the transcription factors (TFs), and their combinatorial interplay [2,3]. TFs regulate the transcription as a response to specific environmental conditions by binding to short degenerate sequence motifs known as transcription factor binding sites (TFBSs) in promoter regions of their target genes and, thereby, enhance or repress gene transcription. Genomic variations, such as single nucleotide polymorphisms (SNPs), define and characterize specific populations or phenotypes and are, hence, used as markers in animal and plant breeding.

Due to the decreasing costs for whole genome sequencing, an increasing number of variants is detected followed by association studies statistically linking SNPs to specific 
traits or diseases. However, the identification of causal variants and the elucidation of their regulatory roles is proceeding at a slow rate $[4,5]$. Today, it is well known that most disease- and trait-associated SNPs are not located within the coding regions of genes but in non-coding regions [6-9]. SNPs that are located in regulatory regions can alter TFBSs leading to a change in the binding affinity of TFs and, in extreme cases, even result in the disruption of a TFBS or the creation of a new TFBS (Figure 1) and, thus, affect gene expression. Such SNPs are referred to as regulatory SNPs (rSNPs) [10-12].

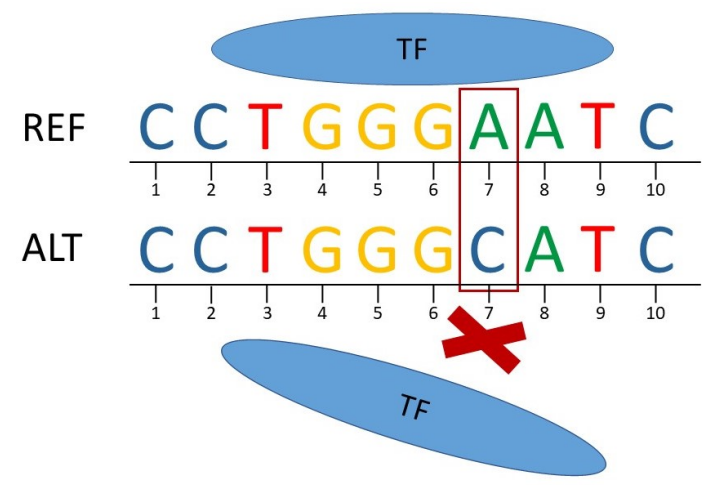

Figure 1. Scheme of the disruption of transcription factor (TF) binding due to a regulatory SNP. The TF can bind to the reference (REF) sequence while it does not bind to the alternate (ALT) sequence (C instead of $A$ at position 7).

The importance of rSNPs has been studied extensively in humans and they are found to have a causal role for numerous traits and diseases [13-16]. A recent review on human rSNPs summarizes different rSNP studies [6]. Due to the great interest in rSNPs, several tools and databases for the analysis of the effects of SNPs on regulatory elements, e.g., TFBSs, have been developed for humans or certain model organisms. Five recent studies are summarized in Table 1, and a comprehensive overview is given in Table S1.

Recently, rSNPs are gaining attention in life sciences and animal breeding since they can be causal for specific traits and diseases and could, hence, serve as new targets for breeding. For this reason, several studies investigated the critical role of rSNPs in agriculturally important species, such as cattle [17-23], pig [24-26], and chicken [27-29]. As these studies were focused on the regulatory role of SNPs for a single trait of interest, they were highly case-specific. Thus, there still exists a lack of systematic analyses of the effects of rSNPs in agricultural species, and, until now, only a few existing tools and databases (DBs) are available for livestock.

MotifbreakR [30] and atSNP [11] are both R packages that principally include all organisms stored in the Bioconductor BSGenome package [31]; however, they require the user to supply the SNP and TFBS data (represented by position weight matrices (PWMs)), and experience in R programming is essential. The Ensembl Variant Effect Predictor (VEP) [32] stores data from experimentally supported and published rSNPs. Due to the lack of experimentally supported data of regulatory elements in livestock, the VEP mainly contains data of regulatory elements and variants for human and mouse. Therefore, the information for livestock stored in the Ensembl VEP is limited to annotations based on the position of the SNP with respect to a gene, e.g., in the upstream region or in the $5^{\prime}$ UTR, excluding effects on TF binding.

In order to address the limited knowledge and information available regarding the crucial functions of rSNPs and their associations with TFBSs in livestock, we systematically carried out an analysis to detect rSNPs and predicted their effects on TF binding for seven agricultural and domestic species (cattle, pig, chicken, sheep, horse, goat, and dog). In particular, we first analyzed the promoter regions (ranging from $-7.5 \mathrm{~kb}$ to $+2.5 \mathrm{~kb}$ ) of all annotated genes and obtained the SNPs within these regions. Secondly, we extracted the flanking sequences for these SNPs and performed a TFBS prediction on the reference as well 
as alternate sequences. Finally, we assigned the identified SNPs to different categories based on their consequences on TF binding (Figure 2) as suggested in [33,34]. To demonstrate our results in a proper way, we developed a database, namely agReg-SNPdb, which stores all predicted regulatory SNPs and their consequences on TF binding for each gene, and we made it accessible via a web interface (https: / / azifi.tz.agrar.uni-goettingen.de/agregsnpdb, (accessed on 16 August 2021)). Furthermore, we performed a literature survey to show that our results are in agreement with previous experimental and in silico studies.

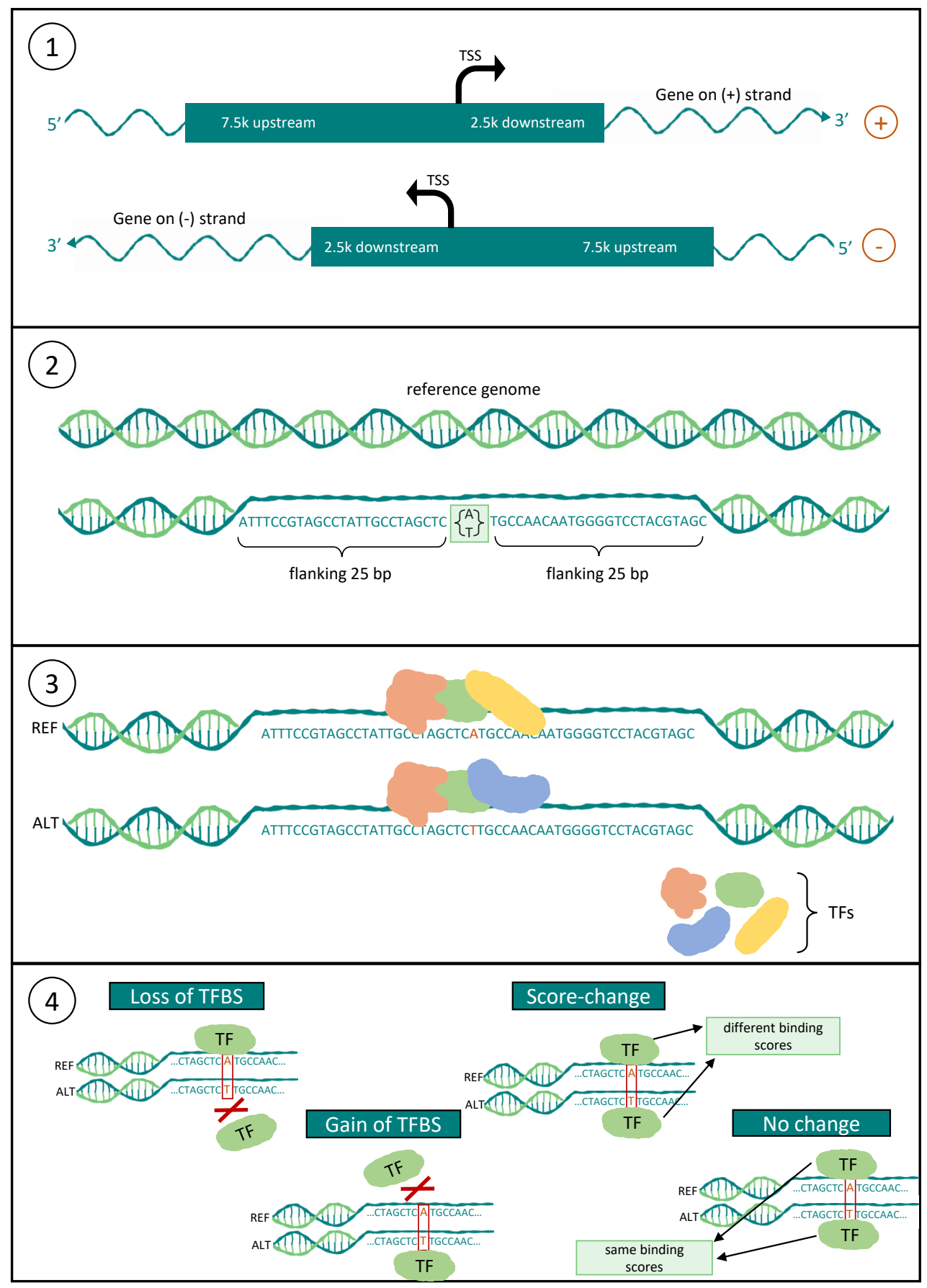

Figure 2. Scheme of the workflow applied for the detection of rSNPs. (1) Definition of the promoter region as $7.5 \mathrm{~kb}$ upstream ( $5^{\prime}$ direction) and $2.5 \mathrm{~kb}$ downstream ( $3^{\prime}$ direction) of the TSS, and extraction of SNPs within this region; (2) extraction of the flanking $25 \mathrm{bp}$ around the SNPs from the reference genome; (3) prediction of the TFBSs for both the reference and alternate sequences; and (4) deriving the consequences for each SNP-TFBS pair. 


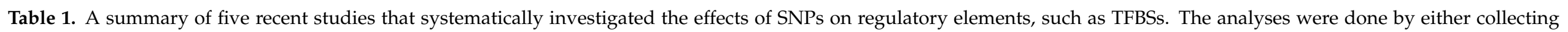
experimentally supported and published data or by predicting the SNP impact on TF binding using prediction tools.

\begin{tabular}{|c|c|c|c|c|c|}
\hline Name & Species & DB/Tool & Website & Characteristics & $\begin{array}{l}\text { Experimentally } \\
\text { Supported Data or } \\
\text { Prediction }\end{array}$ \\
\hline QBiC-Pred [35] & Human & Tool & $\begin{array}{l}\text { http:/ / qbic. } \\
\text { genome.duke.edu } \\
\text { (accessed on } \\
16 \text { August 2021) }\end{array}$ & $\begin{array}{l}\text { - TFBS prediction with regression models } \\
\text { Prediction of changes in TF binding using ordinary least squares and evaluation } \\
\text { of correlation between the predicted binding changes and changes in gene } \\
\text { expression }\end{array}$ & $\begin{array}{l}\text { TFBS } \\
\text { prediction }\end{array}$ \\
\hline INFERNO [37] & Human & Tool & $\begin{array}{l}\text { http:/ /inferno. } \\
\text { lisanwanglab.org } \\
\text { (accessed on } \\
16 \text { August 2021) }\end{array}$ & $\begin{array}{l}\text { - Inferring causal variants from genome-wide association studies (GWAS) within } \\
\text { annotated regulatory regions as enhancers including tissue context } \\
\text { - TFBS prediction with HOMER }\end{array}$ & $\begin{array}{l}\text { TFBS } \\
\text { prediction }\end{array}$ \\
\hline SNP2TFBS [39] & Human & DB & $\begin{array}{l}\text { https://ccg.epfl. } \\
\text { ch//snp2tfbs } \\
\text { (accessed on } \\
16 \text { August 2021) }\end{array}$ & $\begin{array}{l}\text { - DB of human SNPs that affect TFBSs and the prediction of a consequence } \\
\text { - } \quad \text { DB can be downloaded as text files or accessed via the website }\end{array}$ & $\begin{array}{l}\text { TFBS } \\
\text { prediction }\end{array}$ \\
\hline
\end{tabular}




\section{Materials and Methods}

\subsection{Input Data}

The construction of agReg-SNPdb requires: (i) a library of PWMs representing the TFBSs and, for each animal, (ii) a reference genome, (iii) a SNP catalog, and (iv) gene annotations. As a PWM library, we used the non-redundant vertebrate matrices provided by TRANSFAC [40]. The reference genomes, SNP catalogs, and gene annotation files are downloaded from Ensembl [41]. The respective assembly versions are listed in Table 2. The SNP catalog was filtered by discarding all insertions and deletions, keeping only the SNPs. For most genes, more than one transcript isoform was annotated [32], e.g., due to different splicing variants. This ambiguity was kept during the analysis if the positions of the transcription start sites (TSSs) and, hence, the derived promoter regions were different.

Table 2. Assembly versions of the input data, including the reference genome, SNP catalog, and gene annotations. All files were downloaded from Ensembl (release 103).

\begin{tabular}{ccc}
\hline Animal & Assembly Version & Download Date \\
\hline Cattle & ARS-UCD1.2 & 1 March 2021 \\
Pig & Sscrofa11.1 & 9 March 2021 \\
Chicken & GRCg6a & 25 February 2021 \\
Sheep & Oar_rambouillet_v1.0 & 1 March 2021 \\
Horse & EquCab3.0 & 1 March 2021 \\
Goat & ARS1 & 1 March 2021 \\
Dog & CanFam3.1 & 8 March 2021 \\
\hline
\end{tabular}

\subsection{Pipeline}

A general workflow of the detection pipeline is shown in Figure 2. In our previous studies on faba beans [34] and rapeseed [33], we established similar pipelines for the prediction of rSNPs.

\subsubsection{Detection of SNPs within the Promoter Region}

The first step of this analysis was to extract SNPs, which are located within the pre-defined promoter regions. Since there exists no experimentally verified information regarding the exact location of the promoters and in order to overcome inaccuracies in TSS prediction, we chose a large promoter region of $7.5 \mathrm{~kb}$ upstream and $2.5 \mathrm{~kb}$ downstream of the TSS. Similarly large promoter regions were used in previous studies $[10,37,42-48]$. This promoter region can be narrowed by the user during a database search on our website. For all annotated genes, we extracted the SNPs within this region for further analysis by using the function foverlaps of the package data.table in R [49].

\subsubsection{Prediction of TFBSs}

For each SNP lying within a promoter region, we extracted the respective flanking sequence of $25 \mathrm{bp}$ on each side of the SNP resulting in sequences with a total length of $51 \mathrm{bp}$ and the SNP at position 26 (similar flanking sequences were used in $[33,34,43,50]$ ). Sequences with a length of less than $51 \mathrm{bp}$ or sequences with gaps were discarded. After extracting the flanking sequences, we created two sequences per SNP, one with the reference and one with the alternate allele at the SNP position. Both were used as input for the TFBS prediction tool MATCH ${ }^{\mathrm{TM}}$ [51], which scanned the sequences to predict TFBSs using a PWM library from TRANSFAC with specific cutoff values to minimize the false positive rates. If a PWM matched a segment of genomic DNA, this sequence motif was referred to as a (potential) TFBS. As a result, the algorithm provided two scores for each predicted TFBS [40,51]: the matrix similarity score (MSS), measuring the quality of the match regarding the whole PWM sequence, and the core similarity score (CSS), measuring the quality of the match regarding the first five most-conserved consecutive positions of the PWM. Both scores were within the range $[0,1]$, where a score of 1 denoted an exact match of the sequence with the 
PWM [51] measuring the quality of the match and indicating the binding affinity of a TF to the site.

In TRANSFAC, a PWM identifier follows a certain terminology with the structure V\$factorname_version. In our case, each PWM starts with "V\$", which indicates that the PWM originated from a vertebrate TF. The factorname specifies the name of the TF that is binding to the DNA motif. Since there can be several PWMs representing the sequence motif of a specific TF, the version was specified for unique identification $[3,40]$.

\subsubsection{Annotation of Consequences}

For each SNP, we obtained two sets of predicted TFBSs-one for the reference and one for the alternate allele. By comparing these two sets, we manually determined the consequence of a SNP on a TFBS as in our previous studies [33,34]. We differentiated four different consequences: (i) no effect, (ii) change in binding affinity, (iii) loss of TFBS, and (iv) gain of TFBS. We defined two TFBS predictions as the same if their PWMs, positions, and the strand on which they were found were equal for both alleles.

A SNP was considered to have no effect on a TFBS if both scores computed by MATCH $^{\mathrm{TM}}$ were equal for both alleles. A SNP was considered to cause a change in the binding affinity of a TF if the matrix similarity score computed by $\mathrm{MATCH}^{\mathrm{TM}}$ differed for the two alleles. A SNP caused a loss or gain of TFBS if the considered TFBS was only predicted for the reference or alternate sequence, respectively. In this study, we defined an rSNP as a SNP that caused a loss or gain of TFBS or a score-change for at least one TFBS.

\section{Results}

\subsection{Database}

We created the mysql database [52] agReg-SNPdb, which stores (i) general information about the SNPs, such as the ID, chromosomal position and the alleles (table snp_info); (ii) general information about the genes, such as the gene name and chromosomal position (table gene_info); (iii) the table snp_region connecting the tables snp_info and gene_info by storing SNPs and their corresponding target genes together with their genomic position within the promoter region based on the distance to the TSS; and, most importantly, (iv) for each SNP within a promoter region (i.e., for each SNP in table snp_region), we store its consequences based on the predicted TFBS binding potential (table TFBS_results). A summary of the number of entries for each table and animal stored in our database is shown in Table 3.

Table 3. The number of records stored in the database tables snp_info, gene_info, snp_region, and TFBS_results.

\begin{tabular}{ccccc}
\hline & snp_Info & gene_Info & snp_Region & TFBS_Results \\
\hline Cattle & $88,109,946$ & 21,656 & $9,335,814$ & $9,074,371$ \\
Pig & $58,145,647$ & 20,267 & $4,385,724$ & $4,432,047$ \\
Chicken & $20,917,836$ & 16,659 & $3,810,524$ & $3,901,905$ \\
Sheep & $50,164,898$ & 20,359 & $3,216,474$ & $3,205,279$ \\
Horse & $20,331,427$ & 20,499 & $1,585,207$ & $1,713,395$ \\
Goat & $31,331,447$ & 19,658 & $1,987,914$ & $2,015,588$ \\
Dog & $4,725,021$ & 19,960 & 494,691 & 489,292 \\
\hline Total & $273,726,222$ & 139,058 & $24,816,348$ & $24,831,877$ \\
\hline
\end{tabular}

\subsection{Web Interface}

The web interface (https: / / azifi.tz.agrar.uni-goettingen.de/agreg-snpdb, accessed on 16 August 2021) allows users to query the agReg-SNPdb without SQL knowledge and to obtain the requested results either on our website directly or by downloading them as CSV files. The database can be searched by (i) SNP identifiers in the form of rs numbers, (ii) 
SNP positions, (iii) SNP regions in a specified chromosome, or (iv) gene identifiers, i.e., the Ensembl gene stable ID or gene name (Figure 3).

The search results will contain, at maximum, four tables: (1) a table showing general SNP information (table snp_info); (2) a table showing general gene information (table gene_info); (3) a table linking the SNPs to the genes, more specifically to the promoter regions, if they are positioned within a promoter region (table snp_region); and (4) for all rSNPs, a table with the predicted TFBSs overlapping each rSNP, the MATCH ${ }^{\mathrm{TM}}$ scores, and the respective consequence (table TFBS_results) for both alleles. An example output can be seen in Figure 4. In all tables, we provide links to sites with additional information for the SNPs and genes, and, for each PWM, we display the respective sequence logo if desired. Apart from the search site, the complete database tables can be downloaded chromosome-wise on the summary page of the respective animal.

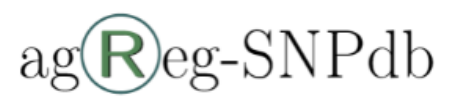

\begin{tabular}{|l|}
\hline$\underline{\text { Home }}$ \\
\hline$\underline{\text { Search }}$ \\
\hline$\underline{\text { Results }}$ \\
\hline$\underline{\text { About }}$ \\
\hline$\underline{\text { Contact }}$ \\
\hline$\underline{\text { Institute }}$ \\
\hline
\end{tabular}

\section{Database search}

\begin{tabular}{|l|}
\hline Species: \\
\hline Search by SNP ID \\
SNP ID (rs number): \\
Search by SNP position \\
Chromosome: \\
Position: \\
Chromosome: \\
start: \\
end: \\
Results are only displayed for regions less than $10 \mathrm{~kb}$. \\
Otherwise the results can only be downloaded. \\
\hline Search by gene (1) \\
Gene: \\
Promoter region (1) \\
\hline
\end{tabular}

Figure 3. Search page of agReg-SNPdb. Search options are (1) by SNP ID, (2) by SNP position, (3) by chromosomal region, and (4) by gene.

\subsection{Statistical Analysis of the Data}

To give a brief overview of the data stored in agReg-SNPdb, we show the distribution of SNPs, genes, and rSNPs in the promoter regions along the chromosomes in an exemplary manner for the species chicken. The distributions for the remaining animals can be found in Figures S2 and S3. The distributions of SNPs and genes along the chromosomes are shown in Figure 5. As expected, the number of SNPs and genes decreased largely with increasing chromosome number and, hence, with decreasing chromosome size. 
SNP information

Show $10 \vee$ entries

\begin{tabular}{|c|c|c|c|c|c|c|c|}
\hline SNP_ID & Chromosome & Position & REF & ALT & Quality & Filter & INFO \\
\hline$\underline{\text { rs41566363 }}$ & 23 & 23277585 & G & C &. &. & ID=51111850;Variant_seq=C;evidence_values=Multiple_observations,Frequency;DDbxref=dbSNP_150:rs41566363;Reference_seq=G \\
\hline
\end{tabular}

Showing 1 to 1 of 1 entries

Gene information

Show $10 \vee$ entries
\begin{tabular}{|l|l|l|l|l|l|}
\hline Name & Chromosome & Strand & txStart & Namearch: \\
\hline ENSBTAG00000020425 & 23 & + & 23277603 & 23337345 \\
\hline Showing 1 to 1 of 1 entries & TFAP2D & t \\
\hline
\end{tabular}

SNP region information

Show $10 \vee$ entries
\begin{tabular}{|l|l|l|l|l|l|l|l|}
\hline SNP_ID & Gene_Name & Chromosome & Strand & txStart & txEnd & Label \\
\hline I541566363 & ENSBTAG00000020425 & 23 & + & 23277603 & 23337345 & inUpstreamPromoterRegion \\
\hline
\end{tabular}

Showing 1 to 1 of 1 entries

Found TFBSs

Explanation of Consequences

Gain of TFBS The TFBS exists only for the 1 (alternative) allele of the SNP

Loss of TFBS The TFBS exists only for the 0 (reference) allele of the SNP

Score-Change The TFBS exists for both alleles but the binding affinity differs as measured by the Core_Similarity_Score and Matrix_Similarity_Score calculated by MATCH

No Change The TFBS exists for both alleles with the same binding affinity

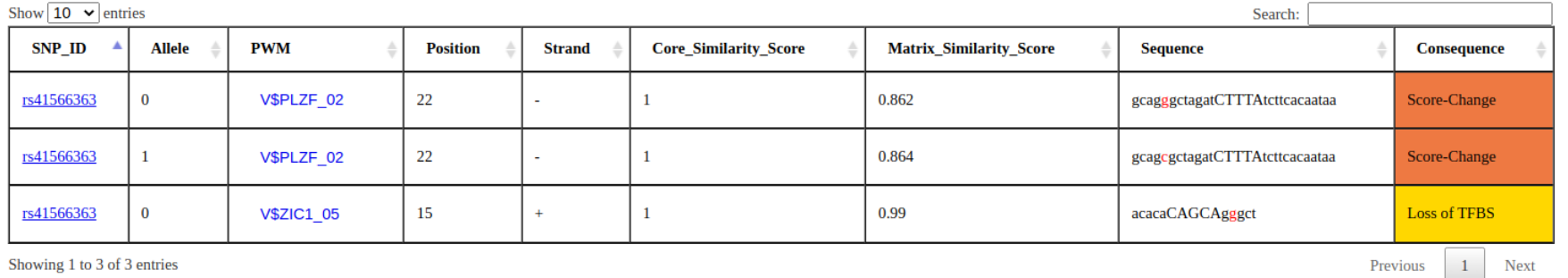

Figure 4. Example of a search result from agReg-SNPdb. The search was performed by the SNP id rs41566363 of cattle. The result tables contain, first, general SNP information; secondly, general gene information; thirdly, information about the SNP region, in particular the promoter region and distance to the TSS; and lastly, the overlapping TFBSs (represented by PWMs) for the SNP with predicted consequences.

Regarding the promoter regions, the number of SNPs in promoters is dependent on the number of genes (Figure 5B) for each chromosome. To overcome this dependency, we calculate the average number of rSNPs per gene in the upstream as well as the downstream promoter region. The average numbers of rSNPs for each chromosome in chicken revealed that most chromosomes had approximately $120 \mathrm{rSNPs}$ per gene, while, on some chromosomes, only very few rSNPs per gene were found (Figure 6). Overall, by dividing the total number of rSNPs by the total number of genes, we identified on average 95.04 rSNPs within the promoter region $(10 \mathrm{~kb})$ of one gene in chicken.

To obtain further insight into the distribution of rSNPs in the promoter regions, we investigated their genomic positions relative to the TSS for the whole promoter region $(-7.5 \mathrm{~kb}$ to $+2.5 \mathrm{~kb})$ and for a smaller section $(-750 \mathrm{bp}$ to $+250 \mathrm{bp})$ for chicken (see Figure 7A,B, respectively; the figures for the remaining species are given in Figures S4). For chicken, we observed a similar finding as in our previous study on rapeseed [33] and as previously shown in rice [53]. While there are few rSNPs in close proximity to the TSS, the number of rSNPs increases with increasing distance to the TSS. Interestingly, in cattle (as well as in dogs), we observed the opposite tendency. Many rSNPs were found around, and especially directly downstream, of the TSS, while the number decreased with the distance to the TSS (the distribution of cattle rSNPs is shown in Figure 8). 
2.4. Chicken
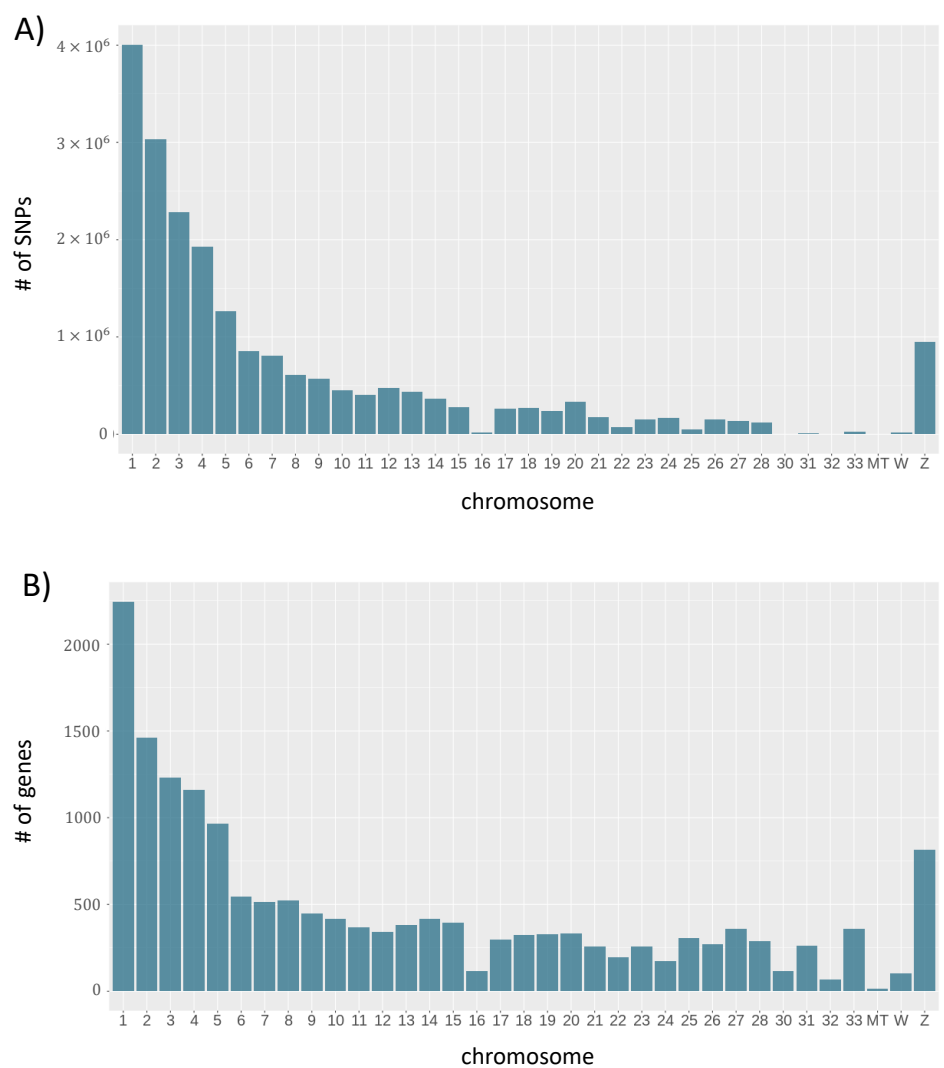

Figure 5. The total number of SNPs and genes for each chromosome of chicken. (A) The number of SNPs per chromosome. (B) The number of genes per chromosome. In total, 20,917,836 SNPs and 16,659 genes were reported. For plotting, the R package ggplot2 [54] was used.

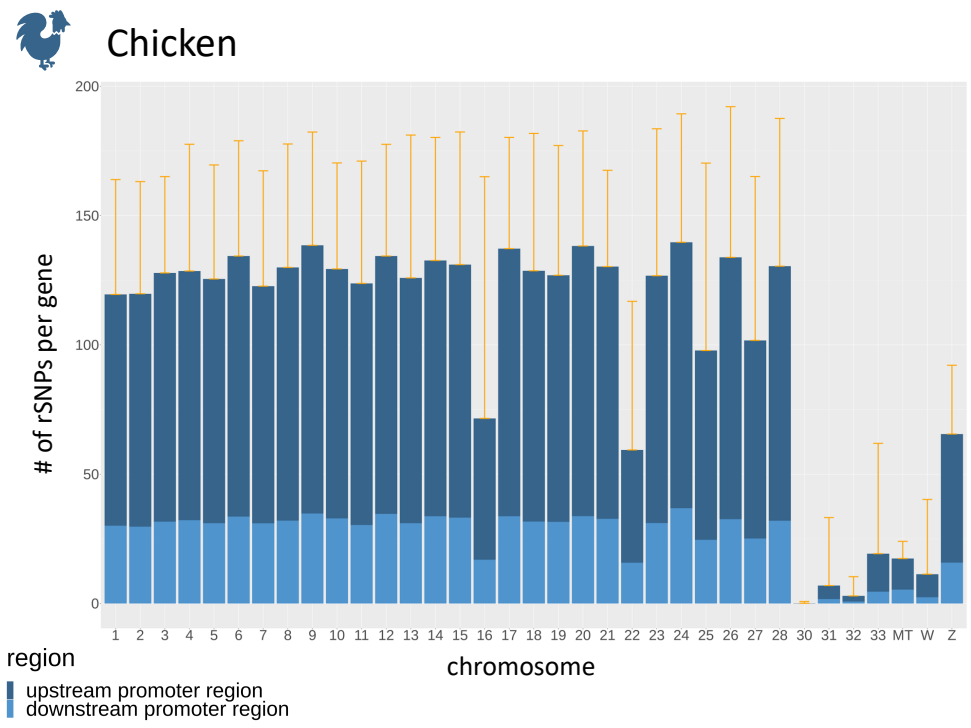

Figure 6. The average number of rSNPs in promoter regions per gene for each chromosome of chicken, divided into upstream and downstream promoters. The orange whiskers denote the mean plus one standard deviation. 


\section{2hicken}



Figure 7. Distribution of the distances between rSNPs and the TSS of chicken. (A) The counts for the whole promoter region $(-7.5 \mathrm{~kb}$ to $+2.5 \mathrm{~kb})$ in $500 \mathrm{bp}$ intervals. The enlargement in (B) shows the proximal promoter region $(-750 \mathrm{bp}$ to $+250 \mathrm{bp})$ in $50 \mathrm{bp}$ intervals. 


\section{Cattle}

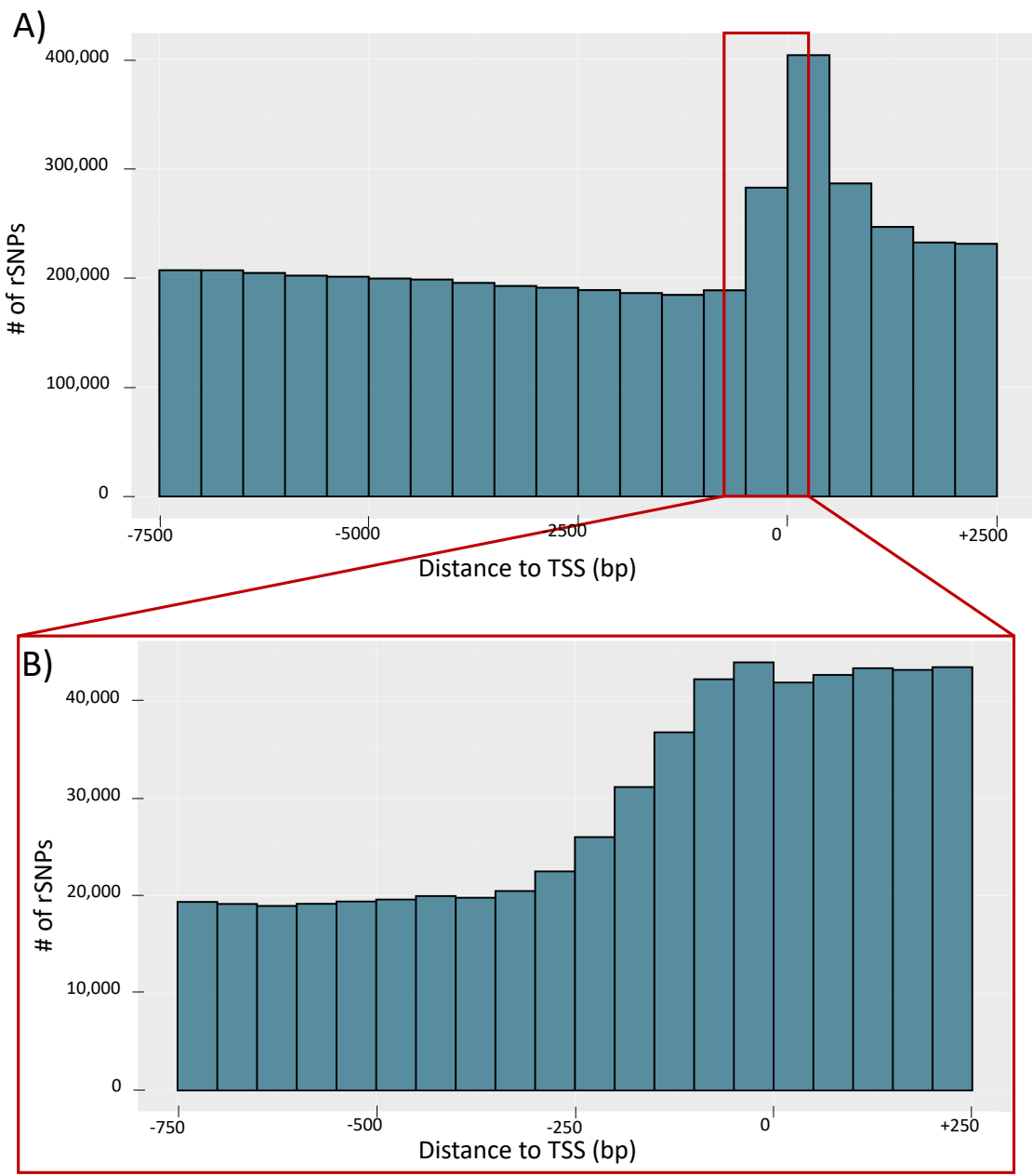

Figure 8. Distribution of the distances between rSNPs and the TSS of cattle. (A) The counts for the whole promoter region $(-7.5 \mathrm{~kb}$ to $+2.5 \mathrm{~kb})$ in $500 \mathrm{bp}$ intervals. The enlargement in $(\mathbf{B})$ shows the proximal promoter region $(-750 \mathrm{bp}$ to $+250 \mathrm{bp})$ in $50 \mathrm{bp}$ intervals.

\section{Biological Validation Based on Case-Studies}

In order to validate the data stored in agReg-SNPdb, we performed literature research and assessed the importance of our findings based on selected published studies, which identified putative rSNPs that are associated with a trait under study and affect TF binding, either by prediction or as evaluated in a biological experiment.

\subsection{Milk Protein and Fat Content in Dairy Cattle}

Lum et al. [23] studied the molecular mechanism of different expression levels of the $ß$-Lactoglobulin (LGB) gene (also known as MBLG or PAEP), which plays an important role in the milk casein, protein, and fat content in dairy cattle. They described one rSNP in the $L G B$ promoter with a $G$ to $C$ conversion $450 \mathrm{bp}$ upstream of the TSS that was found within an activator protein-2 (AP-2) binding site. Measuring the different AP-2 binding affinities with DNase-I footprinting, they measured increased protein binding in the A promoter (G allele).

In our database, we identified the same rSNP (rs41255679, C/G), which was located in the proximal upstream promoter region of PAEP and caused a gain of the AP-2 binding 
site with the G allele (Table 4) [55]. This supports the findings of different studies reporting that AP-2 binding as well as $L G B$ gene expression is enhanced by the G allele and that rs41255679 could be an important regulator of $L G B$ expression [23,55-57].

Table 4. Consequences of SNP rs41255679 (C/G), located upstream of the TSS of the bovine LGB gene. Allele 0 refers to a predicted TFBS in the reference sequence, while allele 1 stands for the alternate allele. A SNP causes a loss of TFBS if the considered TFBS (represented by a PWM) is only predicted for the reference allele. Consequently, a SNP causes a gain of TFBS if the TFBS is only predicted for the alternate allele.

\begin{tabular}{cccc}
\hline SNP ID & Allele & PWM & Consequence \\
\hline rs41255679 & 0 & V\$CTCF_01 & Loss of TFBS \\
rs41255679 & 1 & V\$AP2ALPHA_03 & Gain of TFBS \\
\hline
\end{tabular}

\subsection{Fat-Related Beef Quality Traits in Cattle}

Matsumoto et al. [19] investigated the role of different bovine fat-related genes, including the gene encoding the fatty acid-binding protein 4 (FABP4). Within the FABP4 upstream promoter, they identified two SNPs in linkage disequilibrium (FABP4 g.-295A $>$ G and FABP4 g.-287A $>$ G) that were associated with several fat-related traits, such as the carcass weight and beef marbling score. Using TFSEARCH [58], they predicted TFBSs overlapping the SNPs and altering their binding sites. In agReg-SNPdb, we identified two SNPs within the FABP4 promoter region at a distance of $8 \mathrm{bp}$ to each other and A to $\mathrm{G}$ conversions (respectively, $\mathrm{T}$ to $\mathrm{C}$ conversions, due to the gene's location on the minus strand).

For the first SNP rs110055647, located 123 bp upstream of the TSS, we predicted a loss of TFBS for the Sex-Determining Region Y Protein (SRY) binding site, which is in line with the results of Matsumoto et al. [19]. For the neighboring rs109682576 (-115 bp from the TSS), we did not observe the CCAAT/enhancer-binding protein beta (cEBP/ $\beta$ ) binding site predicted in their study; however, the TFBSs for Zinc finger proteins 333 (ZNF333) and 105 (ZFP105) were lost with the alternate allele, which can be seen as an extension to the results of Matsumoto et al. (Table 5) [19].

Table 5. Consequences of the SNPs rs110055647 and rs109682576 in the bovine FABP4 upstream promoter with a $\mathrm{T}$ to $\mathrm{C}$ conversion. Allele 0 refers to a predicted TFBS in the reference sequence, while allele 1 stands for the alternate allele. A SNP causes a loss or gain of TFBS if the considered TFBS is only predicted for the reference or alternate allele, respectively. A SNP is considered to cause a score-change if the TFBS is predicted on both alleles $(0,1)$ with a difference in the matrix similarity score computed by MATCH ${ }^{\mathrm{TM}}$.

\begin{tabular}{cccc}
\hline SNP ID & Allele & PWM & Consequence \\
\hline rs110055647 & 0,1 & V\$RHOX11_01 & Score-Change \\
rs110055647 & 0 & V\$SRY_Q6 & Loss of TFBS \\
\hline rs109682576 & 0 & V\$ZNF333_01 & Loss of TFBS \\
rs109682576 & 0 & V\$ZFP105_04 & Loss of TFBS \\
\hline
\end{tabular}

\subsection{Chicken Egg Production}

The prolactin $(P R L)$ gene product is considered as an important reproductive hormone involved in diverse biological functions in vertebrates. In laying hens, it is an important regulator of egg production since an increased PRL secretion induces broodiness behaviour [28]. Liang et al. [29] examined the PRL 5' promoter region and, using several populations of Chinese native Yuehuang, Taihe Silkie, and White Leghorn Layer chickens, they identified different rSNPs overlapping the predicted binding sites, including GATA-binding factor 1 (GATA-1), nuclear factor 1 (NF-1), and activator protein 1 (AP-1). Particularly for SNP rs313497646 (A/G conversion, 2048 bp upstream of the TSS), we 
observed the same pattern with respect to TF binding in agReg-SNPdb: only the A allele allows the binding of the NF-1 factor.

Furthermore, it has been shown that the pituitary transcription factor 1 (PIT-1) is an important activator of the PRL gene expression $[28,29,59]$. In agReg-SNPdb, we store a SNP (rs731078272, G/T), located -3086 bp from the TSS and causing a loss of the PIT-1 binding site in the T allele. This result suggests that this SNP might be an important regulator of $P R L$ expression where the T variant could repress $P R L$ expression, which is an important indication for further studies.

\subsection{Fatty-Acid Composition Related Traits in Pigs}

Ballester et al. [24] studied the expression of apolipoprotein (apo-) A-II (APOA2), a protein involved in the triglyceride, fatty acid, and glucose metabolisms, and identified several SNPs associated with $A P O A 2$ gene expression and fatty acid composition traits. Four SNPs were located in the promoter region (rs322246820, rs335066625, rs339777757, and rs333406887), among which they only found one (rs333406887, C/G) influencing a predicted TFBS - in this case, a NF-1 binding site.

Similar to their result, in agReg-SNPdb, we found the SNP rs333406887 overlapping TFBSs, such as the NF-1 binding site. Furthermore, in addition to the reported change in the binding score for NF-1, we can predict several other TFBSs that are affected by this SNP. It causes, for instance, a loss of TFBS for the kruppel-like factor 6 (also called CPBP) and a gain of TFBS for zinc finger protein X-linked (ZFX) (Table 6).

Table 6. Consequences of the SNP rs333406887 (C/G) located -238 bp from the porcine APOA2 TSS. Allele 0 refers to a predicted TFBS in the reference sequence, while allele 1 stands for the alternate allele. A SNP causes a loss or gain of TFBS if the considered TFBS is only predicted for the reference or alternate allele, respectively. A SNP is considered to cause a score-change if the TFBS is predicted on both alleles $(0,1)$ with a difference in the matrix similarity score computed by MATCH ${ }^{\mathrm{TM}}$.

\begin{tabular}{llll}
\hline SNP ID & Allele & PWM & Consequence \\
\hline rs333406887 & 0,1 & V\$NF1_Q6 & Score-Change \\
rs333406887 & 0,1 & V\$AP2ALPHA_03 & Score-Change \\
rs333406887 & 0 & V\$CPBP_Q6 & Loss of TFBS \\
rs333406887 & 1 & V\$ZFX_01 & Gain of TFBS \\
\hline
\end{tabular}

\section{Discussion}

Today, it is widely known that protein-DNA interactions govern the level of gene expression in all higher organisms to a great extent. The binding of TFs to the DNA mainly occurs in the regulatory regions, such as promoters, which are found close to the transcription start of genes [60]. The effect of rSNPs on the binding of TFs has been studied extensively in single case studies in different species, and, for humans, many tools and databases exist to facilitate these analyses (see Tables 1 and S1).

However, there is limited information available for livestock, and, to the best of our knowledge, there is no comparable data source for evaluating the effect of rSNPs. To address this lack of information, we systematically carried out a genome-wide analysis to detect rSNPs and to evaluate their consequences for TF-binding in seven animal species, which can be accessed via a web server. We showed that, by substituting a single base in a predicted TFBS, a SNP can lead to a major change in the binding affinity of the TF and, in an extreme case, even result in the disruption of the TFBS or the creation of a new TFBS.

These predictions can be of great use for scientists who have conducted: (i) an association analysis and want to reveal the underlying mechanisms caused by a SNP being significantly associated with a trait (e.g., in $[19,23,33,34]$ ); (ii) a gene expression experiment and want to identify candidate SNPs influencing the expression rate of a specific gene or a set of genes (e.g., in $[24,29,33])$; or (iii) a combination of both, i.e., an expression quantitative trait locus (eQTL) analysis (e.g., in [17]). 
Even though our predictions are in line with many biologically tested results, as shown in the biological validation in Section 4, we note that the binding affinity of the TFs to the DNA sequence is one of the most important factors for TF binding but might not be sufficient for in vivo binding in higher organisms. Other influencing factors might include the chromatin accessibility, TF concentration, or other enhancing or repressing protein-DNA interactions, such as competitive or cooperative TF binding $[3,39,61]$, which could not be considered in the prediction pipeline.

TF binding often occurs in a complex interplay and also includes cooperation between proximal and distal regulatory elements (promoters and enhancers) [2]. Thus, in addition to the binding of TFs in the proximal promoter regions, regulatory processes via TFDNA interactions are also controlled by distal enhancer regions. Due to the limited knowledge of enhancer regions in livestock species, we could not incorporate these distal regulatory regions.

For our analysis pipeline, we defined a relatively wide promoter region of $7.5 \mathrm{~kb}$ upstream to $2.5 \mathrm{~kb}$ downstream of the TSS. Similarly large promoter regions were defined in previous studies ranging from $10 \mathrm{~kb}$ upstream to $10 \mathrm{~kb}$ downstream of the TSS $[10,37,42-48]$ in order to overcome inaccuracies in the TSS prediction [53] and to ensure the inclusion of the biological promoter. The user has to be aware that the biological promoter region is usually smaller [53], and our website gives the opportunity to filter for smaller, userdefined promoter regions for each single gene. These considered promoter regions and the definition of rSNPs in our study (see Section 2.2.3) led to a relatively large number of rSNPs per gene-for instance, an average of 95.04 rSNPs per gene in chicken.

Interestingly, our results regarding the distribution of genome-wide rSNPs relative to the TSS showed two different patterns. In chicken, pig, sheep, horse, and goat, we observed that the region around the TSS was rather protected from sequence variations (Figure 7) as it was found in previous studies [33,53]. However, the data for cattle and dogs revealed a different picture, and we found an accumulation of SNPs and rSNPs around the TSS (Figure 8). This observation shows that the data stored in public databases, such as Ensembl, can show completely different patterns for different species, which could create biases for specific analyses.

\section{Conclusions}

To the best of our knowledge, agReg-SNPdb is the first database of regulatory SNPs for animal species of agricultural importance. It allows the users to investigate the predicted effect of an allele change on TF binding. The release of the database is an important step toward the understanding of gene regulation in the life sciences. Knowing whether a SNP causes a change in the binding affinity or even disrupts a TFBS or creates a new TFBS can be of predominant importance in order to interpret the results, from, e.g., GWAS experiments, gene expression experiments, or population studies.

The newly gained information can be used to help in genomic selection and marker establishment by identifying possibly causal rSNPs and revealing the underlying regulatory mechanisms of specific traits or diseases. Due to the regular updates of genomes as well as gene and SNP annotations, the database will be updated regularly, and, as future work, we will include several plant species with agricultural importance in agReg-SNPdb.

Supplementary Materials: The following are available online at https:/ / www.mdpi.com/article/10.3 390/biology10080790/s1, Table S1: A comprehensive overview of recent studies that investigated the effects of SNPs on regulatory elements (extension of Table 1), Figure S2: Number of SNPs and genes per chromosomes for all species, Figure S3: The average numbers of rSNPs per gene for each chromosome for all species, Figure S4: Distribution of the distances between rSNPs and the TSS for all species.

Author Contributions: M.G. designed and supervised the research. S.K. and F.H. participated in the design of the study. S.K., F.H., M.G. and A.O.S. conducted the computational and statistical analyses. S.K. performed the biological validation. F.H. created the website. S.K. and F.H. created the database. 
S.K., F.H., and M.G. wrote the final version of the manuscript. M.G. conceived and managed the project. All authors have read and agreed to the published version of the manuscript.

Funding: This research received no external funding.

Institutional Review Board Statement: Not applicable.

Informed Consent Statement: Not applicable.

Data Availability Statement: https:/ / azifi.tz.agrar.uni-goettingen.de/agreg-snpdb (accessed on 16 August 2021).

Acknowledgments: We acknowledge support by the German Research Foundation and the Open Access Publication Funds of the Göttingen University.

Conflicts of Interest: The authors declare no conflict of interest.

\begin{tabular}{|c|c|}
\hline \\
\hline \multicolumn{2}{|c|}{$\begin{array}{l}\text { Abbreviations } \\
\text { The following abbreviations are used in this }\end{array}$} \\
\hline SNP & single nucleotide polymorphism \\
\hline rSNP & regulatory SNP \\
\hline $\mathrm{TF}$ & transcription factor \\
\hline TFBS & transcription factor binding site \\
\hline TSS & transcription start site \\
\hline bp & base pair \\
\hline eQTL & expression quantitative trait locus \\
\hline$\hat{\mathrm{LD}}$ & linkage disequilibrium \\
\hline GWAS & genome-wide association study \\
\hline PWM & position weight matrix \\
\hline SQL & Structured Query Language \\
\hline
\end{tabular}

\section{References}

1. Franco-Zorrilla, J.M.; López-Vidriero, I.; Carrasco, J.L.; Godoy, M.; Vera, P.; Solano, R. DNA-binding specificities of plant transcription factors and their potential to define target genes. Proc. Natl. Acad. Sci. USA 2014, 111, $2367-2372$.

2. Steuernagel, L.; Meckbach, C.; Heinrich, F.; Zeidler, S.; Schmitt, A.O.; Gültas, M. Computational identification of tissue-specific transcription factor cooperation in ten cattle tissues. PLOS ONE 2019, 14, e0216475.

3. Meckbach, C.; Wingender, E.; Gültas, M. Removing background co-occurrences of transcription factor binding sites greatly improves the prediction of specific transcription factor cooperations. Front. Genet. 2018, 9, 189.

4. Hayes, B.J.; Daetwyler, H.D. 1000 Bull Genomes project to map simple and complex genetic traits in cattle: Applications and outcomes. Annu. Rev. Anim. Biosci. 2019, 7, 89-102.

5. Schmitt, A.O.; Aßmus, J.; Bortfeldt, R.H.; Brockmann, G.A. CandiSNPer: A web tool for the identification of candidate SNPs for causal variants. Bioinformatics 2010, 26, 969-970.

6. Degtyareva, A.O.; Antontseva, E.V.; Merkulova, T.I. Regulatory SNPs: Altered Transcription Factor Binding Sites Implicated in Complex Traits and Diseases. Int. J. Mol. Sci. 2021, 22, 6454.

7. Rojano, E.; Seoane, P.; Ranea, J.A.; Perkins, J.R. Regulatory variants: From detection to predicting impact. Briefings Bioinform. 2018, 20, $1639-1654$.

8. Goodswen, S.J.; Gondro, C.; Watson-Haigh, N.S.; Kadarmideen, H.N. FunctSNP: An R package to link SNPs to functional knowledge and dbAutoMaker: A suite of Perl scripts to build SNP databases. BMC Bioinform. 2010, 11, 311.

9. Günther, T.; Schmitt, A.O.; Bortfeldt, R.H.; Hinney, A.; Hebebrand, J.; Brockmann, G.A. Where in the genome are significant single nucleotide polymorphisms from genome-wide association studies located? Omics J. Integr. Biol. 2011, 15, 507-512.

10. Guo, L.; Wang, J. rSNPBase 3.0: An updated database of SNP-related regulatory elements, element-gene pairs and SNP-based gene regulatory networks. Nucleic Acids Res. 2017, 46, D1111-D1116.

11. Zuo, C.; Shin, S.; Keleş, S. atSNP: Transcription factor binding affinity testing for regulatory SNP detection. Bioinformatics 2015, 31, 3353-3355.

12. Macintyre, G.; Bailey, J.; Haviv, I.; Kowalczyk, A. is-rSNP: A novel technique for in silico regulatory SNP detection. Bioinformatics 2010, 26, i524-i530.

13. Buroker, N.E. VEGFA rSNPs, transcriptional factor binding sites and human disease. J. Physiol. Sci. 2014, 64, 73-76.

14. Fang, L.; Ahn, J.K.; Wodziak, D.; Sibley, E. The human lactase persistence-associated SNP- $13910^{*}$ T enables in vivo functional persistence of lactase promoter-reporter transgene expression. Hum. Genet. 2012, 131, 1153-1159. 
15. De Gobbi, M.; Viprakasit, V.; Hughes, J.R.; Fisher, C.; Buckle, V.J.; Ayyub, H.; Gibbons, R.J.; Vernimmen, D.; Yoshinaga, Y.; De Jong, P.; et al. A regulatory SNP causes a human genetic disease by creating a new transcriptional promoter. Science 2006, 312, 1215-1217.

16. Grant, S.F.; Reid, D.M.; Blake, G.; Herd, R.; Fogelman, I.; Ralston, S.H. Reduced bone density and osteoporosis associated with a polymorphic Sp1 binding site in the collagen type I $\alpha 1$ gene. Nat. Genet. 1996, 14, 203.

17. Littlejohn, M.D.; Tiplady, K.; Fink, T.A.; Lehnert, K.; Lopdell, T.; Johnson, T.; Couldrey, C.; Keehan, M.; Sherlock, R.G.; Harland, C.; et al. Sequence-based association analysis reveals an MGST1 eQTL with pleiotropic effects on bovine milk composition. Sci. Rep. 2016, 6, 1-14.

18. Muhaghegh-Dolatabady, M. Single Nucleotide Polymorphism in the Promoter Region of Bovine Interleukin 8 Gene and its Association with Milk Production Traits and Somatic Cell Score of Holstein Cattle in Iran. Iran. J. Biotechnol. 2014, $12,36-41$.

19. Matsumoto, H.; Nogi, T.; Tabuchi, I.; Oyama, K.; Mannen, H.; Sasazaki, S. The SNPs in the promoter regions of the bovine FADS2 and FABP4 genes are associated with beef quality traits. Livest. Sci. 2014, 163, 34-40.

20. Alexandre, P.A.; Gomes, R.C.; Santana, M.H.; Silva, S.L.; Leme, P.R.; Mudadu, M.A.; Regitano, L.C.; Meirelles, F.V.; Ferraz, J.B.; Fukumasu, H. Bovine NR1I3 gene polymorphisms and its association with feed efficiency traits in Nellore cattle. Meta Gene 2014, 2, 206-217.

21. Kühn, C.; Thaller, G.; Winter, A.; Bininda-Emonds, O.R.; Kaupe, B.; Erhardt, G.; Bennewitz, J.; Schwerin, M.; Fries, R. Evidence for multiple alleles at the DGAT1 locus better explains a quantitative trait locus with major effect on milk fat content in cattle. Genetics 2004, 167, 1873-1881.

22. Ordovas, L.; Roy, R.; Pampín, S.; Zaragoza, P.; Osta, R.; Rodriguez-Rey, J.C.; Rodellar, C. The g. 763G> C SNP of the bovine FASN gene affects its promoter activity via Sp-mediated regulation: Implications for the bovine lactating mammary gland. Physiol. Genom. 2008, 34, 144-148.

23. Lum, L.S.; Dovč, P.; Medrano, J.F. Polymorphisms of bovine $\beta$-lactoglobulin promoter and differences in the binding affinity of activator protein-2 transcription factor. J. Dairy Sci. 1997, 80, 1389-1397.

24. Ballester, M.; Revilla, M.; Puig-Oliveras, A.; Marchesi, J.; Castello, A.; Corominas, J.; Fernandez, A.; Folch, J. Analysis of the porcine APOA 2 gene expression in liver, polymorphism identification and association with fatty acid composition traits. Anim. Genet. 2016, 47, 552-559.

25. Ryan, M.T.; Hamill, R.M.; O’Halloran, A.M.; Davey, G.C.; McBryan, J.; Mullen, A.M.; McGee, C.; Gispert, M.; Southwood, O.I.; Sweeney, T. SNP variation in the promoter of the PRKAG3 gene and association with meat quality traits in pig. BMC Genet. 2012, $13,66$.

26. Wyszyńska-Koko, J.; Pierzchała, M.; Flisikowski, K.; Kamyczek, M.; Różycki, M.; Kurył, J. Polymorphisms in coding and regulatory regions of the porcine MYF6 and MYOG genes and expression of the MYF6 gene in m. longissimus dorsi versus productive traits in pigs. J. Appl. Genet. 2006, 47, 131-138.

27. Barkova, O.Y.; Sazanova, K.A.; Fomichev, K.A.; Malewski, T.; Parada, R.; Kawka, M.; Jaszczak, K.; Sazanov, A.A. Associations of new rSNPs with eggshell thickness in Rhode Island layers. Anim. Sci. Pap. Rep. 2013, 31, 165-172.

28. Cui, J.X.; Du, H.L.; Liang, Y.; Deng, X.M.; Li, N.; Zhang, X.Q. Association of polymorphisms in the promoter region of chicken prolactin with egg production. Poult. Sci. 2006, 85, 26-31.

29. Liang, Y.; Cui, J.; Yang, G.; Leung, F.C.; Zhang, X. Polymorphisms of 5 ' flanking region of chicken prolactin gene. Domest. Anim. Endocrinol. 2006, 30, 1-16.

30. Coetzee S.G.; Coetzee, G.A.; Hazelett, D.J. motifbreakR: An R/Bioconductor package for predicting variant effects at transcription factor binding sites. Bioinformatics 2015, 31, 3847-3849.

31. Pagès, H. BSgenome: Infrastructure for Biostrings-based genome data packages and support for efficient SNP representation. $R$ Package 2016, 1, 10-18129.

32. McLaren, W.; Gil, L.; Hunt, S.E.; Riat, H.S.; Ritchie, G.R.S.; Thormann, A.; Flicek, P.; Cunningham, F. The ensembl variant effect predictor. Genome Biol. 2016, 17, 122.

33. Klees, S.; Lange, T.M.; Bertram, H.; Rajavel, A.; Schlüter, J.S.; Lu, K.; Schmitt, A.O.; Gültas, M. In Silico Identification of the Complex Interplay between Regulatory SNPs, Transcription Factors, and Their Related Genes in Brassica napus L. Using Multi-Omics Data. Int. J. Mol. Sci. 2021, 22, 789.

34. Heinrich, F.; Wutke, M.; Das, P.P.; Kamp, M.; Gültas, M.; Link, W.; Schmitt, A.O. Identification of regulatory SNPs associated with vicine and convicine content of Vicia faba based on genotyping by sequencing data using deep learning. Genes 2020, 11, 614.

35. Martin, V.; Zhao, J.; Afek, A.; Mielko, Z.; Gordân, R. QBiC-Pred: Quantitative predictions of transcription factor binding changes due to sequence variants. Nucleic Acids Res. 2019, 47, W127-W135.

36. Shin, S.; Hudson, R.; Harrison, C.; Craven, M.; Keleş, S. atSNP Search: A web resource for statistically evaluating influence of human genetic variation on transcription factor binding. Bioinformatics 2018, 35, 2657-2659.

37. Amlie-Wolf, A.; Tang, M.; Mlynarski, E.E.; Kuksa, P.P.; Valladares, O.; Katanic, Z.; Tsuang, D.; Brown, C.D.; Schellenberg, G.D.; Wang, L.-S. INFERNO: Inferring the molecular mechanisms of noncoding genetic variants. Nucleic Acids Res. 2018, 46, 8740-8753.

38. Guo, L.; Du, Y.; Chang, S.; Zhang, K.; Wang, J. rSNPBase: A database for curated regulatory SNPs. Nucleic Acids Res. 2013, 42, D1033-D1039.

39. Kumar, S.; Ambrosini, G.; Bucher, P. SNP2TFBS-a database of regulatory SNPs affecting predicted transcription factor binding site affinity. Nucleic Acids Res. 2016, 45, D139-D144. 
40. Wingender, E. The TRANSFAC project as an example of framework technology that supports the analysis of genomic regulation. Briefings Bioinform. 2008, 9, 326-332.

41. Yates, A.D.; Achuthan, P.; Akanni, W.; Allen, J.; Allen, J.; Alvarez-Jarreta, J.; Amode, M.R.; Armean, I.M.; Azov, A.G.; Bennett, R.; et al. Ensembl 2020. Nucleic Acids Res. 2020, 48, D682-D688.

42. Ryan, N.M.; Morris, S.W.; Porteous, D.J.; Taylor, M.S.; Evans, K.L. SuRFing the genomics wave: An R package for prioritising SNPs by functionality. Genome Med. 2014, 6, 79.

43. Fu, Y.; Liu, Z.; Lou, S.; Bedford, J.; Mu, X.J.; Yip, K.Y.; Khurana, E.; Gerstein, M. FunSeq2: A framework for prioritizing noncoding regulatory variants in cancer. Genome Biol. 2014, 15, 480.

44. Riva, A. Large-scale computational identification of regulatory SNPs with rSNP-MAPPER. BMC Genom. Biomed Cent. 2012, 13, S7.

45. Kwon, A.T.; Arenillas, D.J.; Hunt, R.W.; Wasserman, W.W. oPOSSUM-3: Advanced analysis of regulatory motif overrepresentation across genes or ChIP-Seq datasets. G3 Genes Genomes Genet. 2012, 2, 987-1002.

46. Coetzee, S.G.; Rhie, S.K.; Berman, B.P.; Coetzee, G.A.; Noushmehr, H. FunciSNP: An R/bioconductor tool integrating functional non-coding data sets with genetic association studies to identify candidate regulatory SNPs. Nucleic Acids Res. 2012, 40 , e139.

47. Ho Sui, S.J.; Mortimer, J.R.; Arenillas, D.J.; Brumm, J.; Walsh, C.J.; Kennedy, B.P.; Wasserman, W.W. oPOSSUM: Identification of over-represented transcription factor binding sites in co-expressed genes. Nucleic Acids Res. 2005, 33, 3154-3164.

48. Stepanova, M.; Tiazhelova, T.; Skoblov, M.; Baranova, A. A comparative analysis of relative occurrence of transcription factor binding sites in vertebrate genomes and gene promoter areas. Bioinformatics 2005, 21, 1789-1796.

49. Dowle, M.; Srinivasan, A.; Gorecki, J.; Chirico, M.; Stetsenko, P.; Short, T.; Lianoglou, S.; Antonyan, E.; Bonsch, M.; Parsonage, H.; et al. Package 'data. table'. Ext. Data Fram. 2019, 1.

50. Xu, Z.; Taylor, J.A. SNPinfo: Integrating GWAS and candidate gene information into functional SNP selection for genetic association studies. Nucleic Acids Res. 2009, 37, W600-W605.

51. Kel, A.E.; Gößling, E.; Cheremushkin, E.; Kel-Margoulis, O.V.; Wingender, E. MATCH: A tool for searching transcription factor binding sites in DNA sequences. Nucleic Acids Res. 2003, 31, 3576-3579.

52. DuBois, P. MySQL; Pearson Education: London, UK, 2008.

53. Triska, M.; Solovyev, V.; Baranova, A.; Kel, A.; Tatarinova, T.V. Nucleotide patterns aiding in prediction of eukaryotic promoters. PLOS ONE 2017, 12, e0187243.

54. Wickham, H. ggplot2: Elegant Graphics for Data Analysis; Springer: New York, NY, USA, 2016.

55. Gambra, R.; Peñagaricano, F.; Kropp, J.; Khateeb, K.; Weigel, K.; Lucey, J.; Khatib, H. Genomic architecture of bovine $\kappa$-casein and $\beta$-lactoglobulin. J. Dairy Sci. 2013, 96, 5333-5343.

56. Schopen, G.; Visker, M.; Koks, P.; Mullaart, E.; Van Arendonk, J.; Bovenhuis, H. Whole-genome association study for milk protein composition in dairy cattle. J. Dairy Sci. 2011, 94, 3148-3158.

57. Kuss, A.; Gogol, J.; Geldermann, H. Associations of a polymorphic AP-2 binding site in the 5'-flanking region of the bovine $\beta$-lactoglobulin gene with milk proteins. J. Dairy Sci. 2003, 86, 2213-2218.

58. Heinemeyer, T.; Wingender, E.; Reuter, I.; Hermjakob, H.; Kel, A.E.; Kel, O.; Ignatieva, E.V.; Ananko, E.A.; Podkolodnaya, O.A.; Kolpakov, F.; et al. Databases on transcriptional regulation: TRANSFAC, TRRD and COMPEL. Nucleic Acids Res. 1998, 26, 362-367.

59. Nelson, C.; Albert, V.R.; Elsholtz, H.P.; Lu, L.; Rosenfeld, M.G. Activation of cell-specific expression of rat growth hormone and prolactin genes by a common transcription factor. Science 1988, 239, 1400-1405.

60. Meckbach, C.; Tacke, R.; Hua, X.; Waack, S.; Wingender, E.; Gültas, M. PC-TraFF: Identification of potentially collaborating transcription factors using pointwise mutual information. BMC Bioinform. 2015, 16, 400.

61. Hughes, T.R. A Handbook of Transcription Factors; Springer: Dordrecht, The Netherlands, 2011; Volume 52. 